\title{
Potential of Nonlinear Ultrasonic Indicators for Nondestructive Testing of Concrete
}

\author{
C. Payan, V. Garnier, and J. Moysan \\ Laboratoire de Caractérisation Non Destructive, Université de la Méditerranée, IUT Aix-Provence, Avenue Gaston Berger, \\ 13625 Aix-en-Provence Cedex, France
}

Correspondence should be addressed to C. Payan, cedric.payan@univmed.fr

Received 7 October 2009; Revised 15 February 2010; Accepted 14 April 2010

Academic Editor: Patrice Rivard

Copyright () 2010 C. Payan et al. This is an open access article distributed under the Creative Commons Attribution License, which permits unrestricted use, distribution, and reproduction in any medium, provided the original work is properly cited.

\begin{abstract}
In the context of a growing need for safety and reliability in Civil Engineering, acoustic methods of nondestructive testing provide answers to a real industrial need. Linear indicators (wave speed and attenuation) exhibit a limited sensitivity, unlike nonlinear ones which usually have a far greater dynamic range. This paper illustrates the potential of these indicators, and evaluates its potential for in situ applications. Concrete, a structurally heterogeneous and volumetrically, mechanically damaged material, is an example of a class of materials that exhibit strong multiple scattering as well as significant elastic nonlinear response. In the context of stress monitoring in pre-stressed structures, we show that intense scattering can be applied to robustly determine velocity changes at progressively increasing applied stress using coda wave interferometry and thereby extract nonlinear coefficients. In a second part, we demonstrate the high sensitivity of nonlinear parameters to thermal damage as regard with linear ones. Then, the influence of water content and porosity on these indicators is quantified allowing to uncouple the effect of damage from environmental or structural parameters.
\end{abstract}

\section{Introduction}

The present paper is devoted to the study of the potential applications of techniques derived from nonlinear acoustics, applied to the nondestructive testing of concrete. Nonlinear methods have been considered, because nonlinearity indicators exhibit large dynamic ranges, which can be used in homogenous media [1]. Their dynamic range is often ten times greater than that of linear parameters.

One of the major challenges for non-destructive evaluation in civil engineering is the need for indicators which can faithfully provide relevant in situ information. The characteristics of concrete, a structural material, evolve with time, as a function of structural (composition, damage, ect.) and environmental (water content, temperature, prestress, ect.) parameters. Being a strongly inhomogeneous medium, which naturally has microcracks, it has a strongly nonlinear behaviour, characteristic of so-called "nonclassical" materials, as revealed in the 1990's for rocks [2] or more recently for granular materials [3]. Researchers have described strongly nonlinear phenomena in the context of dynamic tests. Since these effects are not accounted for by Murnaghan's [4] "classical" theory, which involves a development to orders greater than 1 of the behaviour law, the authors introduced the term "non-classical". Indeed, rocks are made of grains and microcracks, the assembly of which is characterised by an elastic modulus $M_{0}$. Contacts between the grains, and friction in the microcracks represent a range of elastic elements which may control the assembly's nonlinear behaviour when traversed by a wave of amplitude $\Delta \varepsilon[5]$

$$
M(\varepsilon, \dot{\varepsilon})=M_{0}[1-\beta \varepsilon-\alpha(\Delta \varepsilon+\operatorname{sign}(\dot{\varepsilon}) \varepsilon)]
$$

where $\beta$ characterises the classical nonlinearity, $\alpha$ the nonclassical nonlinearity, and $\varepsilon$ is the instantaneous strain.

With respect to wave propagation, the term $\beta$ represents the variation of the wave's velocity resulting from the strain state, in the case of both static and dynamic loading. This phenomenon is known as acoustoelasticity in homogeneous materials $[4,6]$. In the case of strongly inhomogeneous 
and/or damaged (rocks, concrete, ect.) materials, and nonclassical nonlinearity $\alpha$ enables hysteresis and discrete memory phenomena to be represented [2].

As a result, these materials present a resonant frequency shift when increasing excitation amplitude. Under two harmonic sources (frequencies $f_{1}$ and $f_{2}$ with $f_{2}>f_{1}$ ), the nonlinearity generates new frequency components called harmonics $\left(2 f_{1}, 3 f_{1}, \ldots\right.$ and $\left.2 f_{2}, 3 f_{2} \ldots\right)$ and interactions or side bands $\left(f_{2}-f_{1}, f_{2}+f_{1}, f_{2}+2 f_{1} \ldots\right)$.

These indicators have already been used for the Non Destructive Testing (NDT) of concrete. Mechanical damage has been widely discussed. Fatigue damage as been monitored by nonlinear resonance methods using flexural [5] and compressional [7] modes. Compressional damage as been monitored by nonlinear interaction [8] and harmonic generation [9]. The setting of concrete has also been monitored using this type of method $[10,11]$. These indicators have recently proved efficient in detecting chemical attack of concrete such as Alcali/Silica reaction (ASR) [12, 13]. The influence of water saturation on the classical nonlinear parameter $(\beta$ in $(1))$ has been demonstrated by harmonic generation $[14]$.

\section{Motivations}

In the present study, we propose to investigate several aspects of the problems currently encountered in civil engineering.

The first of these is related to the evaluation of stress in the inaccessible, pre-stressed cables embedded in concrete structures. Because of the inaccessible nature of these cables, we have concentrated our efforts on the influence of stress on concrete, the outer surface of which remains accessible. For that purpose, we study the classical nonlinear properties of concrete, the parameter $\beta$, which relates the wave speed dependence with stress state, using an original approach based on the analysis of the "coda" of ultrasound signals. The main advantage of this approach is that the relative velocity variation which can be detected are very small $(<1 / 10000)$ and thereby allows to detect very small stress variation.

The second aspect is related to reduce the threshold damage detection. In this context, the sensitivity analysis of the non-classical nonlinear parameter $\alpha$, in the presence of thermal damage is achieved. We compare the evolution dynamics of the linear and nonlinear parameters.

Having demonstrated the strong sensitivity of this indicator to damage, the third aspect is related to the study of the influence of concrete's environmental and structural parameters (water content porosity) on its nonlinear non classical response. The aim of this analysis is to separate the influence of these two effects with the ultimate goal to apply this method on real concrete structures.

\section{Evaluation of the Prestressed Condition}

The variation of the velocity of ultrasonic waves resulting from static loading has been well understood in isotropic homogeneous materials since the 1960's. Described by classical expressions of the 2 nd order (linear elasticity) as a function of $\varepsilon$, Hughes and Kelly [6] derived the expressions for the velocity of elastic waves in an infinite, stressed material, using Murnaghan's [4] theory at the third order. By assuming a static uniaxial stress in direction 1, where $(1,2,3)$ forms an orthonormal basis, Egle and Bray [15] have shown that the third order elastic constants: $l, n$ and $m$, called Murnaghan's constants, can be derived from a simple linear relationship between the second order elastic constants (Young's Modulus and Poisson's ratio) and the quantities $L_{i j}$ defined by $d V_{i j} / V_{i j}^{0}=L_{i j} d \varepsilon$. With $\varepsilon=d u / d x$ giving the strain in direction $1, V_{i j}$ is the velocity of the waves propagating in direction $i$ and polarised in direction $j$. $V_{i j}^{0}$ represents the velocity of the waves in the reference (unstressed) state. To summarise, by assuming the second order elastic constants to be known, we need 3 velocity measurements in direction 2 (perpendicular to the direction of stress) in order to evaluate the 3 third order constants.

We have studied a promising method which enables the scattering properties of concrete to be put to advantage, thereby avoiding the need for reference state measurements (velocity and distance). Inspired from geophysics, this method, based on the analysis of the coda of transmitted signals, is found to have considerable potential for in situ applications.

The term coda, generally used in reference to the finale of a musical composition, is used in geophysics to describe the end of seismograms recorded during ground movements. The seismic coda is composed of multiple waves, which are reflected and propagated through the Earth's crust, and recorded at the surface. In concrete, the same phenomena occur on a smaller scale. The geophysicists began taking an interest in the coda of seismic waves in the early 1970's. The simple, but ingenious idea was then to exploit all of the information available in seismograms. Until then, they were interested only in the arrival of direct waves, thus losing most of the information contained in the signals. The first author to study seismic codas was Aki [16], who showed that they result from the diffusion of waves in the Earth's mantle. The method was refined several times, by Poupinet et al. [17] and Roberts et al. [18], up until the most recent progress made by Snieder, referred to as "Coda Wave Interferometry" (CWI) [19] (Figure 1).

Velocity variations are not apparent at the beginning of the signal (Figure 1(b)), but are clearly visible in the coda (Figure 1(c)). In this observation window, the delay between the two signals is obtained by cross-correlation, by measuring the temporal position of the maximum of this function (Figure 1(d)). By taking the average of the delays over all of the sliding windows, the relative variation in velocity is found using $d V / V^{0}=-\langle t / \tau\rangle$, where $t$ is the position of the centre of the observation window. This method allows us to study, at high resolution and for different levels, the velocity variations induced by a quasi-static stress.

We used a hydraulic press (MTS 318.25), into which we introduced a concrete sample with a diameter of $75 \mathrm{~mm}$ and a length of $160 \mathrm{~mm}$ (Figure 2). Preliminary tests enabled Young's modulus $(42.39 \mathrm{GPa})$ and Poisson's ratio $(0.21)$ to be estimated. Strain gauges were glued to the test sample 


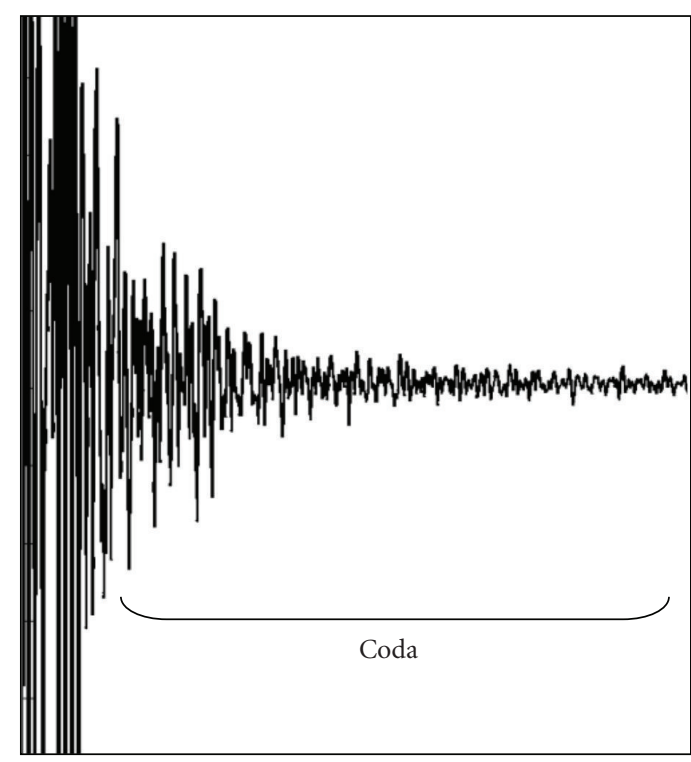

(a)

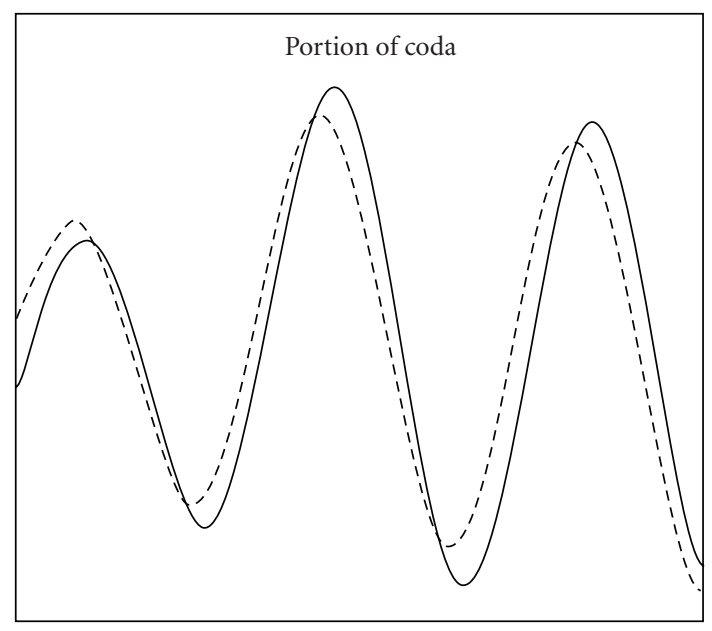

(c)

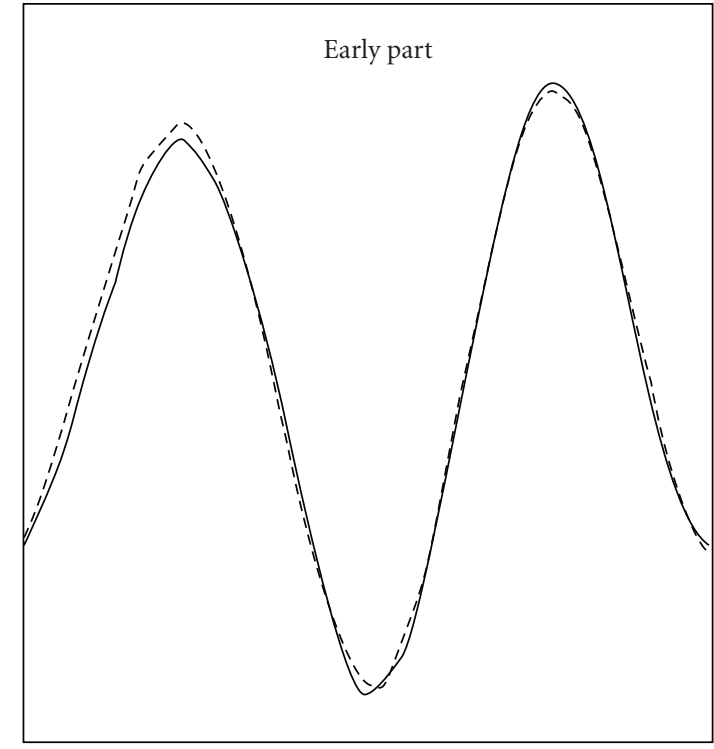

(b)

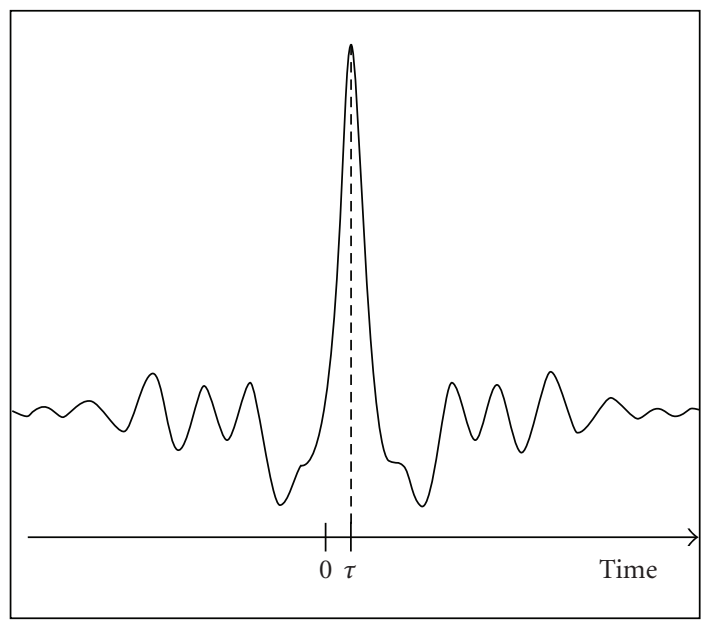

(d)

Figure 1: Implementation of the CWI method. Solid line: reference signal. Dotted line: signal under stressed conditions. (a) Typical signals recorded on our sample. (b) Early part of the signal. (c) Portion of the coda. (d) Cross-correlation function.

in order to monitor the strain in the load direction (direction 1). Compressional and shear ultrasonic transducers $(500 \mathrm{kHz})$ were used, and held in place by means of a springloaded device which provides a constant load. These pull in direction 2, using an impulse. In this way, the CWI method allows us to determine the quantities $d V_{2 j} / V_{2 j}^{0}(j=1 \cdots 3)$ and as the strain is known, we can derive the values of $L_{2 i}$ using $d V_{2 j} / V_{2 j}^{0}=L_{2 j} d \varepsilon$. All of the results are represented in Figure 3. It is important to note that the CWI method enables relative variations in velocity much smaller than $1 / 1000$ to be detected. The nonlinear parameter $\beta$ (1) can be expressed as a function of Murnaghan's constants $l, m, n$ [20] and the nonlinear parameter obtained from the relation $\beta=3 / 2+$ $(l+2 m) /(\lambda+2 \mu)$. The strongly negative values shown in (Table 1) are similar to those determined in rocks $[20,21]$, and several orders of magnitude greater than those found with homogeneous materials $[15,20]$. For the purposes of comparison, the nonlinear parameter $\beta$ can be derived by studying the material's behaviour during a static test. Our result is in agreement with those found in the literature for concrete with the same composition [22].

The following section is devoted at studying the sensitivity of the nonlinear non classical parameter $\alpha$ to thermal dama.

\section{Sensitivity of Nonlinear Indicators to Thermal Damage}

In civil engineering, the problem of monitoring tunnels after a fire, or of drums of stored radioactive waste, is currently debated. Following the study of classical nonlinearity, we 


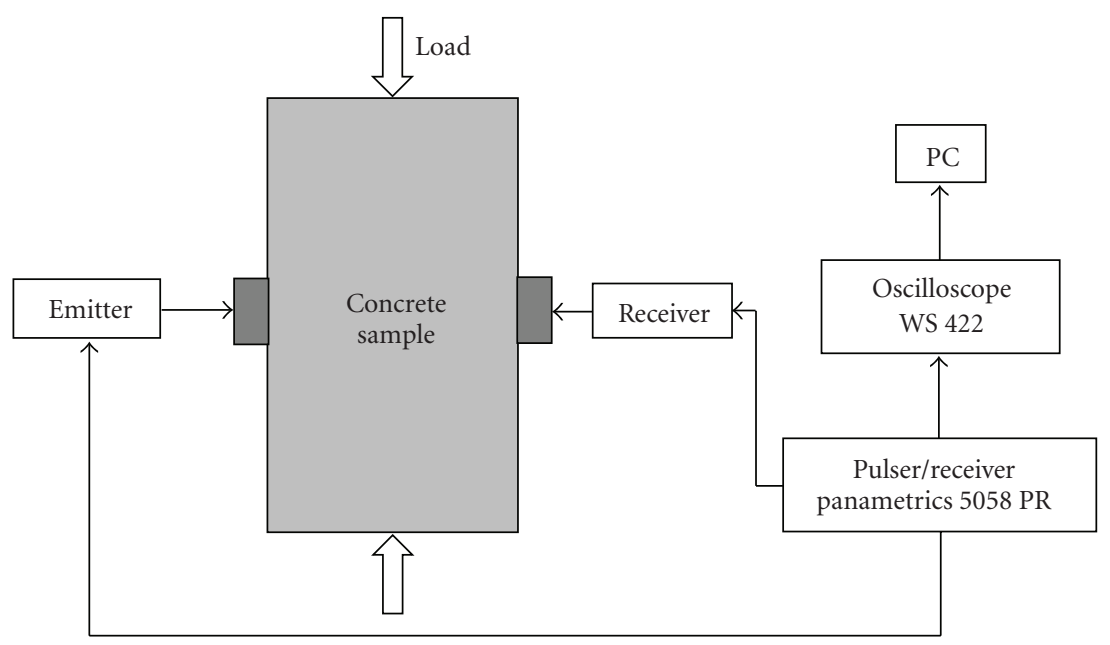

FIGURE 2: Experimental setup

TABLE 1: Third order elastic constants and nonlinear parameter determined for concrete and some other materials from literature.

\begin{tabular}{lcccc}
\hline & $l(\mathrm{GPa})$ & $m(\mathrm{GPa})$ & $n(\mathrm{GPa})$ & $\beta$ \\
\hline Iron [10, 15] & -348 & -1030 & 1100 & 420 \\
Pyrex glass [10, 15] & 14 & 92 & -6600 & -7.3 \\
Granite [15, 16] & -3371 & -6742 & -84900 & -441 \\
Sandstone [15, 16] & -97800 & -99400 & $n / a$ & -9600 \\
Concrete from [17] & $n / a$ & $n / a$ & $-1813 \pm 3.4 \%$ & -139 \\
Concrete (present) & $-3007 \pm 2.8 \%$ & $-2283 \pm 1.2 \%$ & & $-157 \pm 1.9 \%$ \\
\hline
\end{tabular}

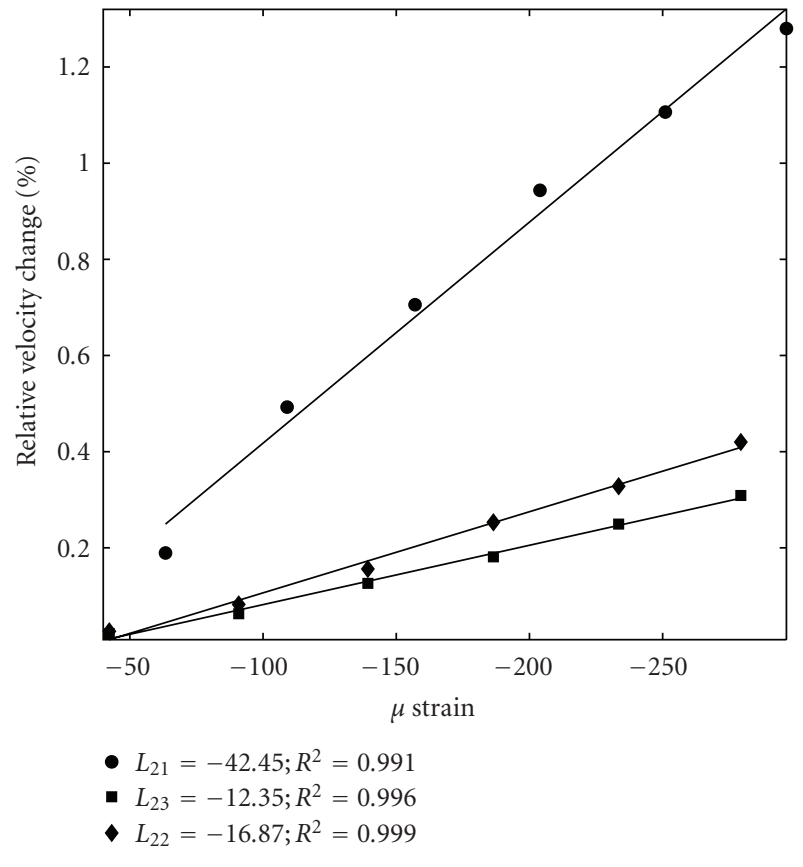

FIGURE 3: Relative velocity variations as a function of strain.

now focus on the non-classical nonlinearity ( $\alpha$ parameter in (1)) of concrete affected by thermal damage. For that, we compare the sensitivity of a linear indicator, that is, the velocity of ultrasound waves, with the nonclassical nonlinear parameter $\alpha$.

We used four identical samples, of parallelepiped geometry with dimensions $10 \times 10 \times 5 \mathrm{~cm}^{3}$. The first sample was kept as a reference, the second was heated to $120^{\circ} \mathrm{C}$, the third to $250^{\circ} \mathrm{C}$, and the fourth to $400^{\circ} \mathrm{C}$ following a heating/cooling cycle, based on rates of change of the order of $1^{\circ} \mathrm{C} /$ minute, in order to avoid any risk of bursting the samples.

For the linear method, a flight-time measurement is carried out in order to evaluate the velocity $V_{p}$ of the compressional waves. The NRUS (Nonlinear Resonant Ultrasound Spectroscopy) method was used to derive the nonlinear parameter $\alpha$. This is achieved by putting the sample into resonance, at successively higher amplitudes. In non-classical materials, the resonance frequency reduces linearly as a function of the driving amplitude (Figure 3 ) and the nonlinear parameter is obtained by simplifying equation (1) to a first order expression [23]

$$
\frac{f_{0}-f}{f_{0}}=\alpha \Delta \varepsilon,
$$

where $f_{0}$ is the "linear" resonance frequency, that is, that obtained for small amplitudes, and $f$ is the resonance frequency measured at successively higher amplitudes $\Delta \varepsilon$. In the present study, we used ultrasonic transducers (Panametrics V1012 $100 \mathrm{kHz}$ ) for both emission and reception, such that the measured values are not absolute, but can 


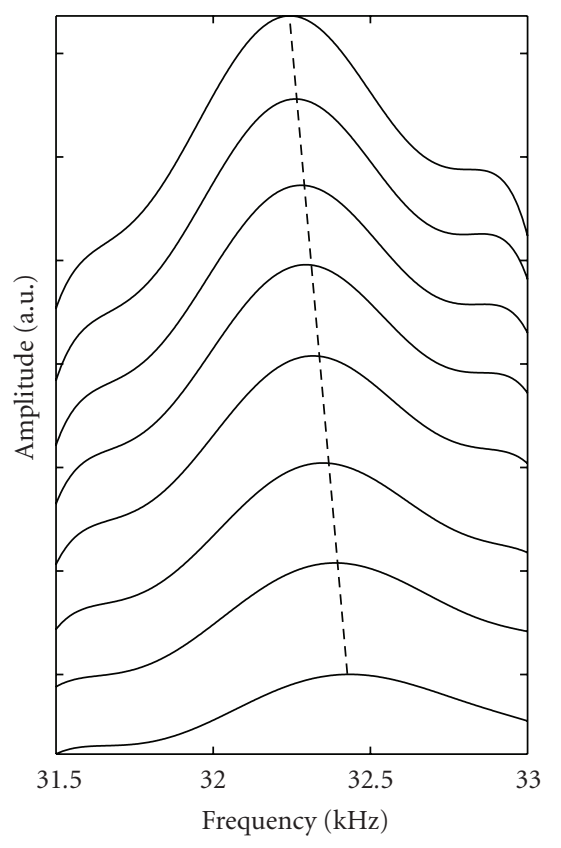

FIGURE 4: NRUS experiment for the $120^{\circ} \mathrm{C}$ damaged sample.

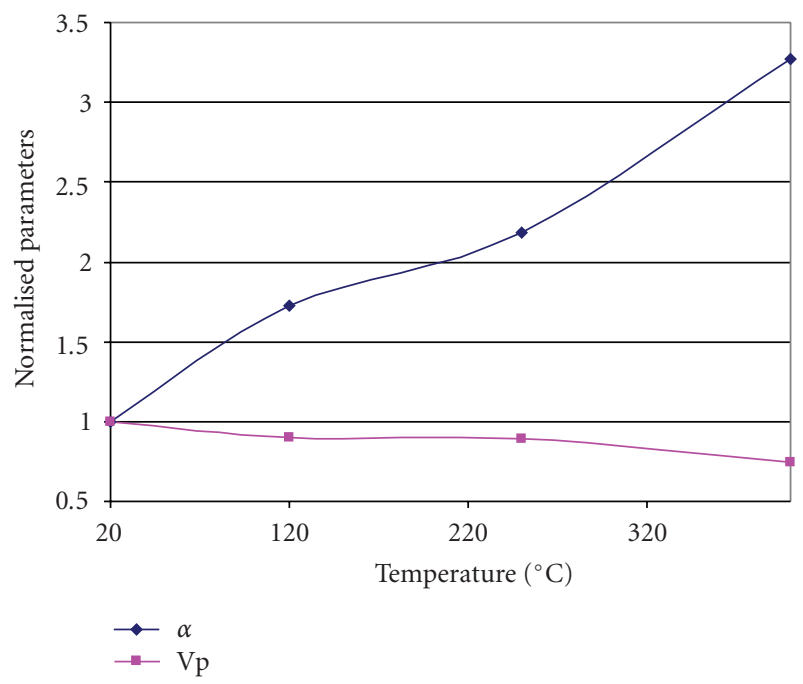

FIgURE 5: Relative variation of the nonlinear parameter $\alpha$ and of the velocity $V_{p}$ of pressure waves as a function of the damage temperature.

nevertheless be compared with each other. The linearity of the detection chain was verified using a steel sample with dimensions similar to those of our concrete samples.

We observe that there is a visible shift in resonance frequency (Figure 4 ). The nonlinear parameter, derived from the slope of the dotted straight line in Figure 4 (2), has a dynamic range ten times greater than that of the velocity of the ultrasonic waves (Figure 5).

The next section is aimed at quantifying the contribution of environmental and structural concrete parameters on the nonlinear $\alpha$ parameter.

\section{Influence of Water Content and Porosity on the Nonlinear Parameter}

In order to study the influence of water content and porosity on the nonlinear response of concrete, we used 5 series of 6 parallelepiped samples $\left(12 \times 25 \times 50 \mathrm{~cm}^{3}\right)$. Each series had a different water-to-cement ratio, and thus a different porosity (determined by the water-to-cement ratio W/C). These samples were prepared with water saturations of 0 , $40,60,80$ and $100 \%$. At saturations $0 \%$ and $100 \%$, the full sample sets were respectively dried or saturated. For the intermediate states, only part of the samples in any given series was prepared with a water saturation of 40,60 or $80 \%$.

The wave interaction method is used to extract the nonlinear parameter $\alpha$. The principle is based on the use of two waves: a high-energy low frequency $\left(f_{1}\right)$ wave and a high frequency $\left(f_{2}\right)$ wave. The low frequency perturbs the material, whilst the high frequency "inspects" the same material, thus conveying information related to its nonlinearity. If both waves propagate in a material governed by a law of the type in (1), the high frequency is modulated in amplitude by the low frequency, thus producing new frequencies [24]: $f_{2}-f_{1}, f_{2}+f_{1}, f_{2}+2 f_{1}, f_{2}-2 f_{1}, \ldots$ The nonlinear parameter can then be derived from the ratio between the amplitudes of these new frequencies and those of the waves from which they were produced $\left(f_{1}\right.$ and $\left.f_{2}\right)$

In the case of the present study (Figure 6), the high frequency $(250 \mathrm{kHz})$ is produced by a function generator, which is used to continuously drive a Panametrics V1012 transducer. Signal reception is achieved using an identical transducer, connected to an oscilloscope. The low frequency is generated by mechanical impact. A steel ball is released from a constant height, thus exciting all of the sample's resonance frequencies, and a low frequency tranducer connected to the oscilloscope records the associated signal and synchronised the acquisition of data. As the resulting frequency spectrum is complex (see Figure 6 FFT(C1)), it is difficult to extract the amplitudes and frequencies resulting from this interaction. Nevertheless, Van Den Abeele et al. [24] have shown that, instead of using an amplitude ratio, the nonlinear parameter $\alpha$ could be obtained using an energy ratio

$$
\alpha=\frac{W_{N L}}{W_{f_{1}} W_{f_{2}}},
$$

where $W_{N L}, W_{f 1}, W_{f 2}$ are respectively the energies contained in all of the spectral bands generated by the interaction, at the low and high frequencies (see Figure 6).

The first measurement campaigns dealt with the saturation states of $0 \%$ and $100 \%$. The results are presented in Figure 7. The first conclusion that can be drawn is that the water content has a significant influence on the nonlinear parameter $\alpha$, with an average variation of $100 \%$ between the two states. The second conclusion is that porosity has only a minor influence on the concrete's nonlinear behaviour, at least over the usual W/C range $(0.3<W / C<0.65)$. This observation allows us to average the full set of results obtained with the samples, for a given level of saturation. These results are compiled in Figure 8. 


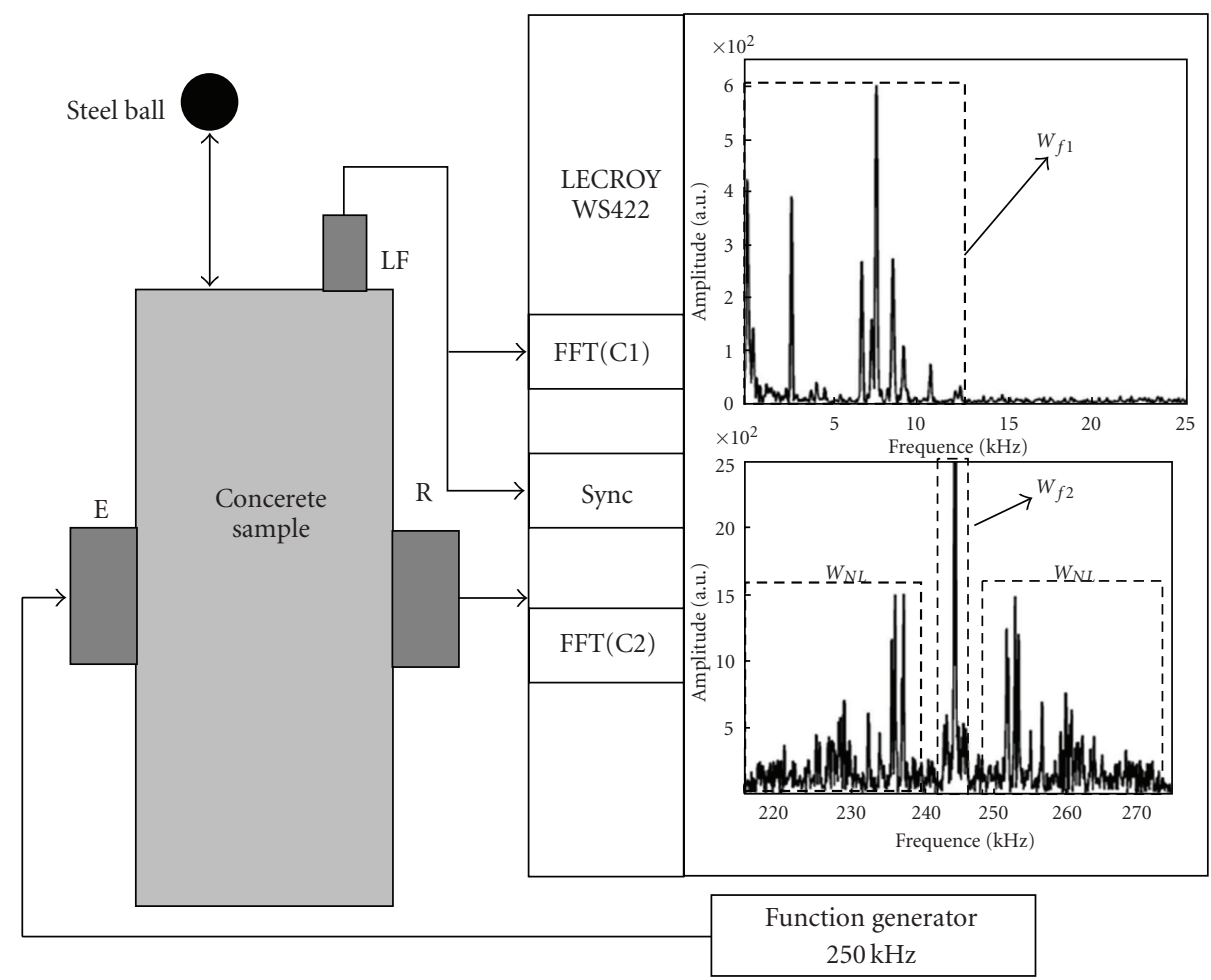

Figure 6: Experimenatl setup. FFT (C1) and FFT (C2) are respectively the typical frequency spectrums of LF and HF obtained in experiments. FFT (C2) illustrates the interaction of HF with LF.

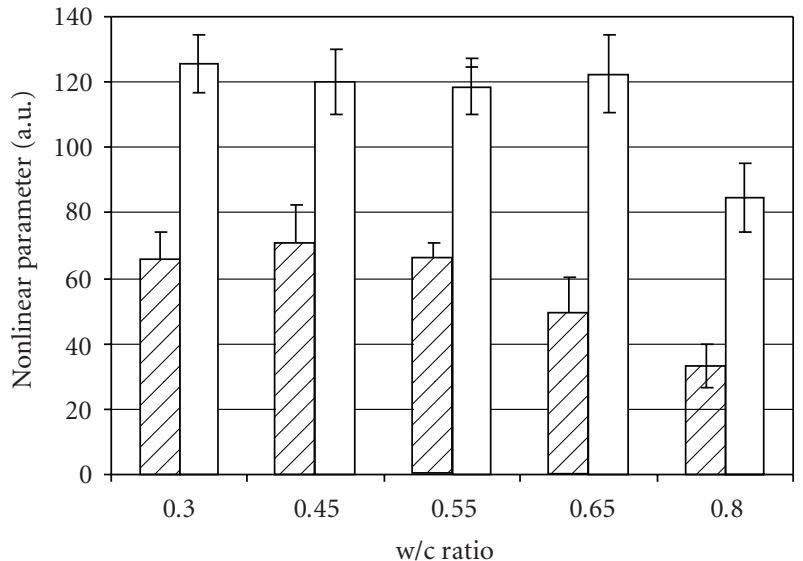

$\square 100 \%$

$\square 0 \%$

Figure 7: Nonlinear parameter measured at $0 \%$ and $100 \%$ saturation, as a function of the water to cement ratio.

To the best of our knowledge, no former studies have derived the dependence of the nonlinear $\alpha$ parameter on the water content of concrete. Only two other studies have investigated this dependence $[25,26]$ in rocks. The results are highly variable, according to the type of rock. However, one trend can be extracted from our results: the sudden drop in nonlinear behaviour is similar to that observed for chalk, but very different to that observed in sandstone or limestone.

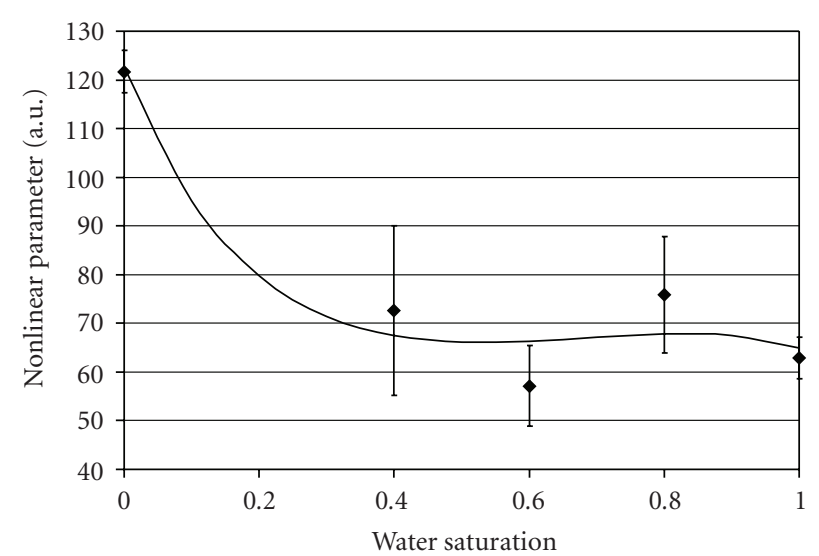

Figure 8: Dependence of the nonlinear parameter on the degree of water saturation

\section{Discussion and Conclusions}

In this paper, we demonstrate the high potential of nonlinear acoustic techniques, applied to the in situ NDT of concrete.

We propose a robust and accurate method for monitoring the stressed state of concrete, thereby removing the need to measure a distance. The in situ measurement of absolute stress level appears to be difficult at the present time. The CWI method can nevertheless be used to monitor stress in pre-stressed concrete structures. The simplicity with which this method can be implemented, its high sensitivity, and the 
limited quantity of instrumentation needed, favour its use for the in situ monitoring of civil engineering structures.

The high sensitivity of the nonlinear $\alpha$ parameter to thermal damage enables perceptible variations to be observed, at the very outset of a damage process, thereby reducing the detection threshold. Although this large dynamic range is positive, the role of potentially influential parameters, such as water content or the concrete's porosity, should also be queried with the perspective of in situ monitoring. The method proposed in this section, for the extraction of the nonlinear parameter $\alpha$, is difficult to transpose into an in situ context. Indeed, it appears problematic to identify the resonance modes of a real structure. In addition, the in situ generation of waves of sufficient amplitude is likely to be challenging.

In the last section, we have quantified the influence of water content and porosity on the nonlinear $\alpha$ parameter of concrete, thus allowing us to decouple the effects resulting from damage, resulting for example from water content. Regarding the evolution of the nonlinearity with water saturation from $20 \%$ to $100 \%$, the nonlinear parameter can be considered as constant in this range. So, nonlinear acoustics should not be used for water saturation characterization in concrete. Nevertheless, concrete structures are exposed to atmospheric conditions, between $30 \%$ and $100 \%$ of water saturation. Since this non destructive method is highly sensitive to damage, it is a great candidate for health monitoring of concrete structures. In addition, the proposed method is highly promising for applications involving the in situ NDT of concrete structures, using a high amplitude, low frequency wave produced by a mechanical impact, which propagates throughout the structure. It could also be possible to make use of natural excitations of the structure like wind or traffic.

\section{Acknowledgments}

These studies were conducted in the context of two French national projects (ACTENA and SENSO) supported by the French Research National Agency, funded by Electricité De France (EDF) with the technical support of the Laboratoire de Mécanique et d'Acoustique (CNRS, UPR-7051). Authors would thank Paul Johnson (Los Alamos National Laboratory, USA) for comments and discussions. C. Payan gratefully thank Julie Baret for helpful technical support.

\section{References}

[1] P. B. Nagy, "Fatigue damage assessment by nonlinear ultrasonic materials characterization," Ultrasonics, vol. 36, no. 1-5, pp. 375-381, 1998.

[2] R. A. Guyer and P. A. Johnson, "Nonlinear mesoscopic elasticity: evidence for a new class of materials," Physics Today, vol. 52, pp. 30-36, 1999.

[3] P. A. Johnson and X. Jia, "Nonlinear dynamics, granular media and dynamic earthquake triggering," Nature, vol. 437, no. 7060, pp. 871-874, 2005.

[4] F. D. Murnaghan, Finite Deformations of an Elastic Solid, John Wiley \& Sons, New York, NY, USA, 1951.
[5] K. Van Den Abeele and J. De Visscher, "Damage assessment in reinforced concrete using spectral and temporal nonlinear vibration techniques," Cement and Concrete Research, vol. 30, no. 9, pp. 1453-1464, 2000.

[6] D. S. Hughes and J. L. Kelly, "Second-order elastic deformation of solids," Physical Review, vol. 92, no. 5, pp. 1145-1149, 1953.

[7] M. Bentahar, H. El Aqra, R. El Guerjouma, M. Griffa, and M. Scalerandi, "Hysteretic elasticity in damaged concrete: quantitative analysis of slow and fast dynamics," Physical Review B, vol. 73, no. 1, Article ID 014116, pp. 1-10, 2006.

[8] K. Warnemuende and H.-C. Wu, "Actively modulated acoustic nondestructive evaluation of concrete," Cement and Concrete Research, vol. 34, no. 4, pp. 563-570, 2004.

[9] A. A. Shah, Y. Ribakov, and S. Hirose, "Nondestructive evaluation of damaged concrete using nonlinear ultrasonics," Materials and Design, vol. 30, no. 3, pp. 775-782, 2009.

[10] J.-C. Lacouture, P. A. Johnson, and F. Cohen-Tenoudji, "Study of critical behavior in concrete during curing by application of dynamic linear and nonlinear means," Journal of the Acoustical Society of America, vol. 113, no. 3, pp. 1325-1332, 2003.

[11] K. Van Den Abeele, W. Desadeleer, G. De Schutter, and M. Wevers, "Active and passive monitoring of the early hydration process in concrete using linear and nonlinear acoustics," Cement and Concrete Research, vol. 39, pp. 426-432, 2009.

[12] A. Kodjo, P. Rivard, F. Cohen-Tenoudji, and J. L. Gallias, "Evaluation of damages due to alkali-silica reaction with nonlinear acoustics techniques," POMA, vol. 7, no. 1, Article ID 045003, 10 pages, 2009.

[13] J. Chen, A. R. Jayapalan, J.-Y. Kim, K. E. Kurtis, and L. J. Jacobs, "Rapid evaluation of alkali-silica reactivity of aggregates using a nonlinear resonance spectroscopy technique," Cement and Concrete Research, vol. 40, no. 6, pp. 914-923, 2010.

[14] D. Zhou, X. Liu, and X. Gong, "Water content diagnostics of concrete using nonlinear acoustics means," in Proceedings of the 17th World Conference on Nondestructive Testing, October 2008.

[15] D. M. Egle and D. E. Bray, "Measurement of acoustoelastic and 3rd-order elastic-constants for rail steel," Journal of the Acoustical Society of America, vol. 60, no. 3, pp. 741-744, 1976.

[16] K. Aki, "Analysis of coda of local earthquakes as scattered waves," Journal of Geophysical Research, vol. 74, no. 2, pp. 615$631,1969$.

[17] G. Poupinet, W. L. Ellsworth, and J. Frechet, "Monitoring velocity variations in the crust using earthquake doublets: an application to the Calaveras fault, California ( USA)," Journal of Geophysical Research, vol. 89, no. 7, pp. 5719-5731, 1984.

[18] P. M. Roberts, W. S. Phillips, and M. C. Fehler, "Development of the active doublet method for measuring small velocity and attenuation changes in solids," Journal of the Acoustical Society of America, vol. 91, no. 6, pp. 3291-3302, 1992.

[19] R. Snieder, A. Grêt, H. Douma, and J. Scales, "Coda wave interferometry for estimating nonlinear behavior in seismic velocity," Science, vol. 295, no. 5563, pp. 2253-2255, 2002.

[20] P. A. Johnson and P. N. J. Rasolofosaon, "Nonlinear elasticity and stress-induced anisotropy in rock," Journal of Geophysical Research B, vol. 101, no. 2, pp. 3113-3124, 1996.

[21] P. Rasolofosaon, B. Zinszner, and P. A. Johnson, "Propagation of elastic waves in nonlinear materials: survey of laboratory results on rock and geophysical applications," Oil \& Gas Science and Technology, vol. 52, no. 6, pp. 585-608, 1997.

[22] I. E. Shkolnik, "Effect of nonlinear response of concrete on its elastic modulus and strength," Cement and Concrete Composites, vol. 27, no. 7-8, pp. 747-757, 2005. 
[23] K. E.-A. Van Den Abeele, J. Carmeliet, J. A. Ten Cate, and P. A. Johnson, "Nonlinear elastic wave spectroscopy (NEWS) techniques to discern material damage, part II: single-mode nonlinear resonance acoustic spectroscopy," Research in Nondestructive Evaluation, vol. 12, no. 1, pp. 31-42, 2000.

[24] K. E.-A. Van Den Abeele, P. A. Johnson, and A. Sutin, "Nonlinear Elastic Wave Spectroscopy (NEWS) techniques to discern material damage, Part I: nonlinear wave modulation spectroscopy (NWMS)," Research in Nondestructive Evaluation, vol. 12, no. 1, pp. 17-30, 2000.

[25] K. E.-A. Van Den Abeele, J. Carmeliet, P. A. Johnson, and B. Zinszner, "Influence of water saturation on the nonlinear elastic mesoscopic response in Earth materials and the implications to the mechanism of nonlinearity," Journal of Geophysical Research B, vol. 107, no. 6, 11 pages, 2002.

[26] P. A. Johnson, B. Zinszner, P. Rasolofosaon, F. CohenTenoudji, and K. Van Den Abeele, "Dynamic measurements of the nonlinear elastic parameter $\alpha$ in rock under varying conditions," Journal of Geophysical Research B, vol. 109, no. 2, Article ID 10129, pp. 1-12, 2004. 

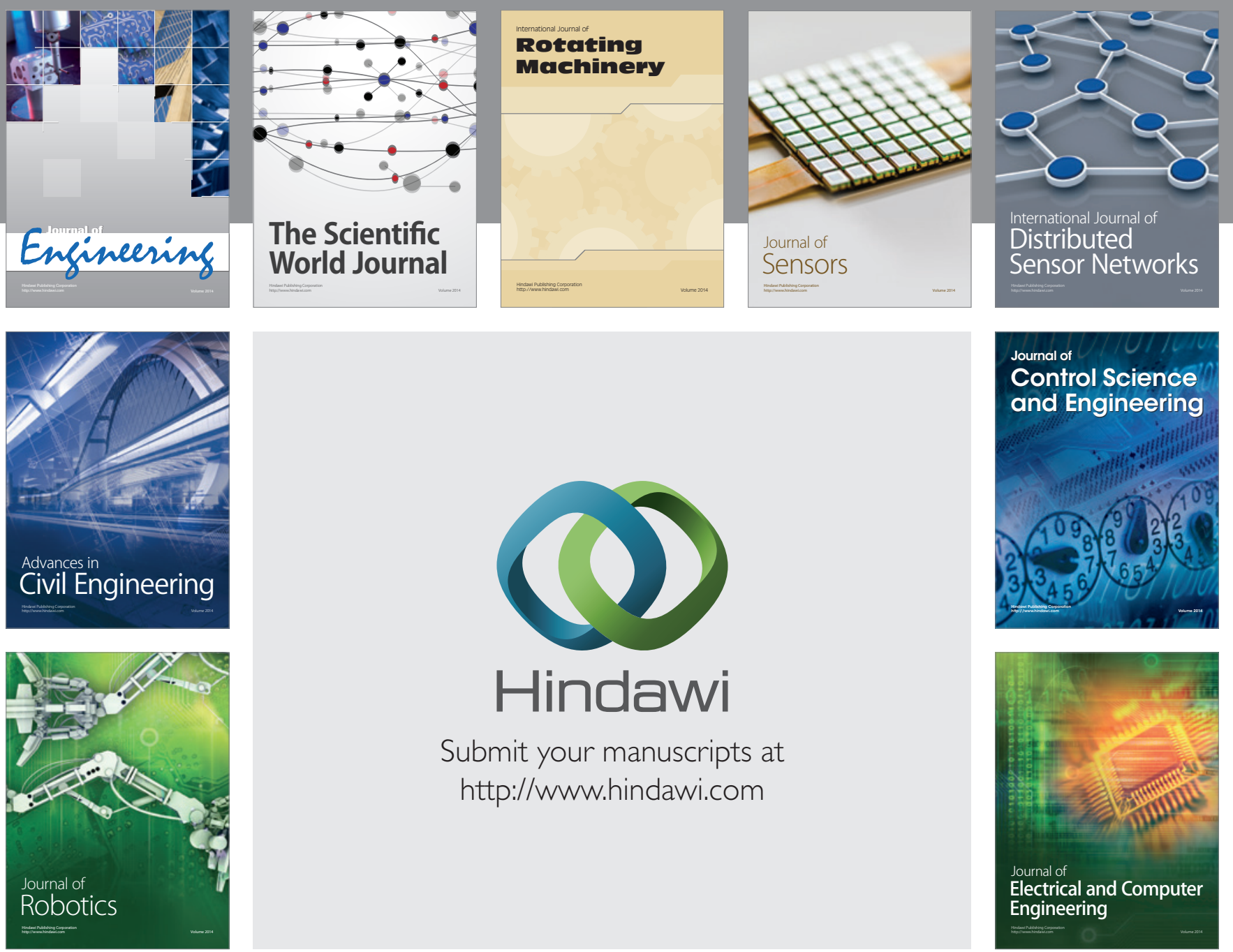

Submit your manuscripts at

http://www.hindawi.com
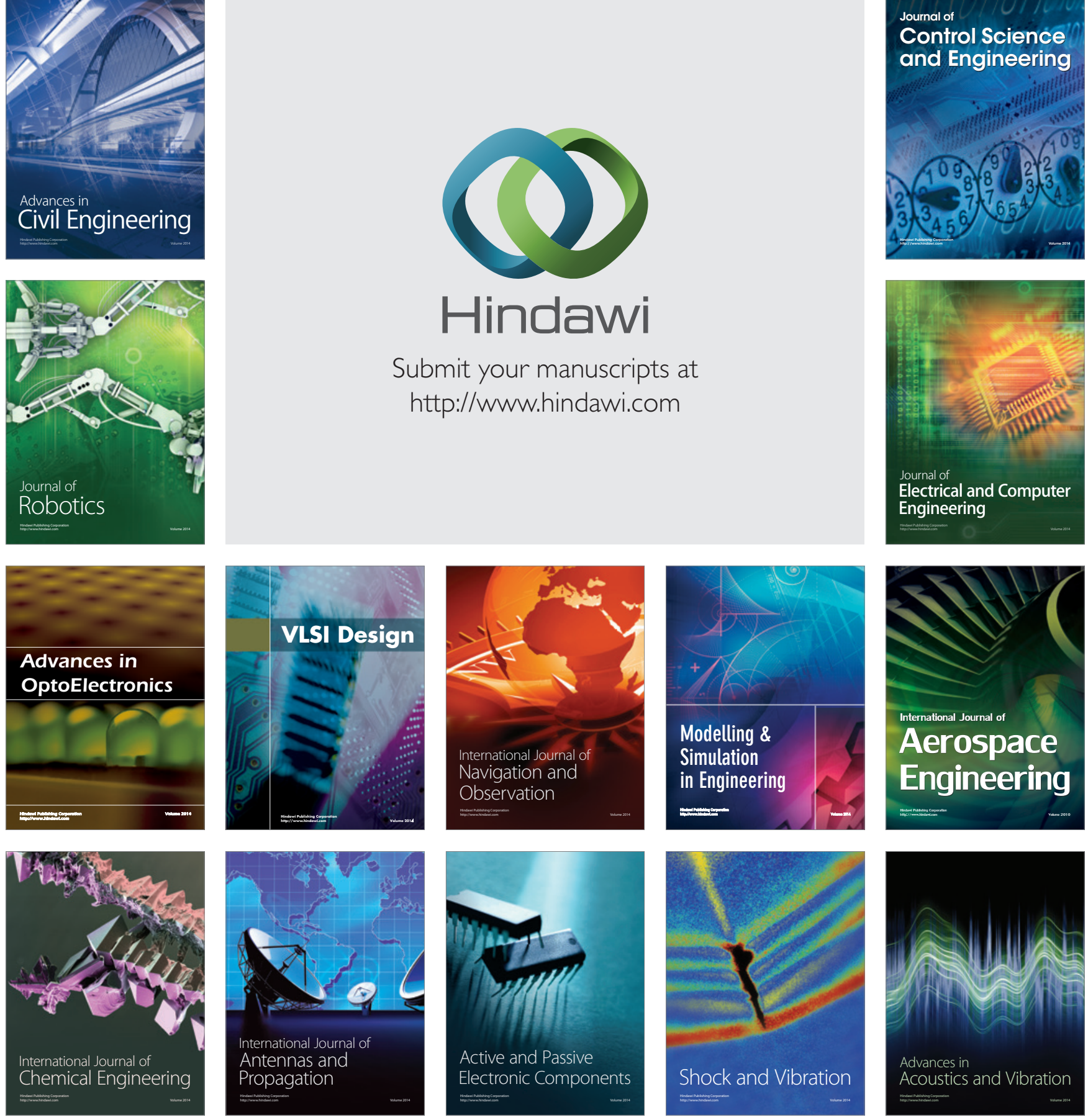